\title{
La glucemia dos horas post-estímulo fue mejor que la glucemia en ayunas para predecir el riesgo de muerte a largo plazo
}

Glucose tolerance and mortality: comparison of WHO and American Diabetes Association diagnostic criteria. The DECODE study group. European Diabetes Epidemiology Group. Diabetes Epidemiology: Collaborative analysis Of Diagnostic criteria in Europe. Lancet 1999: 354:617-21.

El test de tolerancia a la glucosa (glucemia 2 hs. postestímulo) y no la glucemia de ayuno identifica mejor a los individuos con riesgo aumentado de muerte, asociada con hiperglucemia.

Objetivo

1) Identificar individuos asintomáticos con mayor riesgo de mortalidad relacionada a hiperglucemia. 2) Comparar los criterios diagnósticos de Diabetes Mellitus (DM) de la Asociación de Diabetes Americana (ADA, de EE.UU) con los de la Organización Mundial de la Salud (OMS). 3) Establecer un criterio diagnóstico de DM en relación a las complicaciones macrovasculares (principal causa de muerte en DM 2)

\section{Diseño}

Estudio multicéntrico con seguimiento prospectivo para evaluación del riesgo de muerte asociado a hiperglucemia. Seguimiento promedio de 7.3 años.

Lugar

Datos de 13 estudios de cohorte europeos.

\section{Pacientes}

Se realizó un Test de Tolerancia a la Glucosa con 75 gr. (TTOG) a 25364 individuos asintomáticos de 30 a 89 años (18 048 hombres y 7316 mujeres). Se correlacionó en los mismos, la glucemia basal y la de las 2 hs. (G2h) con el riesgo de muerte durante un período de seguimiento medio de 7.3 años.

\section{Evaluación de los factores pronósticos}

Se evaluaron como variables el sexo y las glucemias basales y G2hs. Se utilizó análisis multivariado ajustando por edad, sexo y centro.

\section{Medición de resultado principal}

Los datos obtenidos permitieron establecer 3 diferentes niveles de riesgo según si su TTOG fuera normal $(\mathrm{N})$, o bien demostrara intolerancia a la glucosa (IGT) o diabes (DBT).

\section{Resultados principales}

Hubo 2121 muertes acumuladas. Comparado con individuos $\mathrm{N}$, los varones con DM (criterio ADA: glucemia de ayuno $>126 \mathrm{mg} / \mathrm{dl}$ ) presentaron riesgo relativo de muerte de 1.81 (IC 95\% 1.49 a 2.20 ) y las mujeres de 1.79 (1.18 a 2.69). Para quienes evidenciaron glucemia alterada de ayuno (GAA) (criterio ADA: glucemia $110-126 \mathrm{mg} / \mathrm{dl})$ el riesgo fue de 1.21 (IC95\% $1.05 \mathrm{a} 1.41)$ en varones y 1.08 (IC95\% 0.70 a 1.66) en mujeres. En los que presentaron $D M$ según el criterio de la OMS (glucemias $>200 \mathrm{mg} / \mathrm{dl}$ a 2 hs.), el riesgo fue de 2.02 (IC95\% 1.66 a 2.46) en hombres y de 2.77 (IC95\% 1.96 a 3.92) en mujeres. Para IGT (criterio OMS: glucemias 140 a $200 \mathrm{mg} / \mathrm{dl} 2 \mathrm{hs}$. post-sobrecarga) el riesgo fue de 1.51 (IC95\% 1.32 a 1.72) en hombres y 1.6 (IC95\% 1.22 a 2.10$)$ en mujeres. Dentro de cada clasificación de glucemia basal (normal: <110, GAA: $110-126$ y DM: $>126 \mathrm{mg} / \mathrm{dl}$ ) la mortalidad aumentaba significativamente si se consideraban los incrementos de la G2h. Sin embargo la inversa no se constató (Gráfico 1). Los $\mathrm{N}$ presentaron un riesgo de muerte de 6\%, los DM (por hiperglucemia de ayuno) 16\%, los DM por G2h elevada15\% y los IGT 12\% (Gráfico 2).

Gráfico 1 - Indices de mortalidad en relación con la glucemia basal y glucemia 2-horas post-sobrecarga en individuos que no se sabían diabéticos.

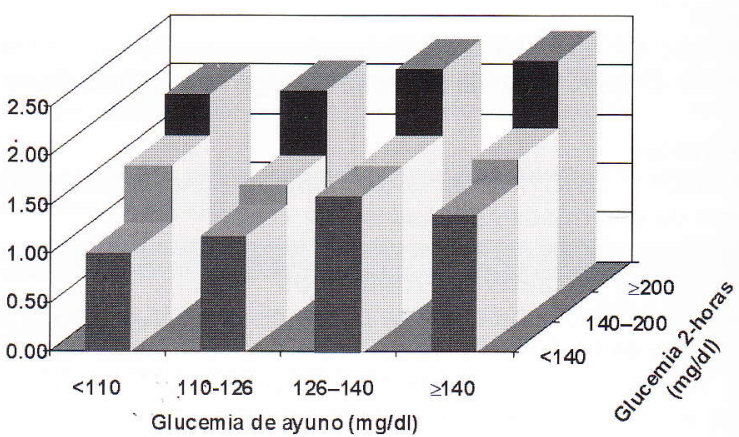

Decode Study Group. Lancet 1999;354:617-2

Gráfico 2 - DECODE: la mortalidad incrementa con el aumento de la glucemia 2-horas post-sobrecarga

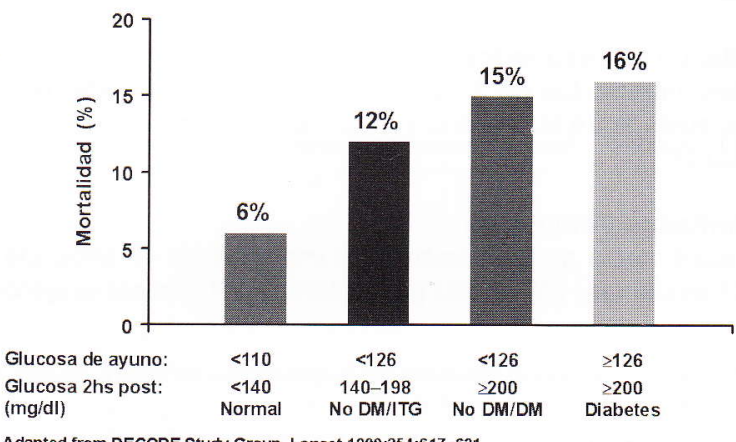

\section{Conclusiones}

La glucemia en ayunas solamente no identifica a individuos con un aumento de muerte relacionado a la hiperglucemia. EI TTOG provee información pronóstica adicional, y permite la detección de individuos con IGT, que son los que más riesgo tienen dentro de cada nivel de glucemia en ayunas.

Fuente de financiamiento: no referida.

\section{Comentario}

Este importante trabajo, junto a otros de menor envergadura, ha demostrado que la asociación de alteraciones glucémicas y el riesgo de muerte está claramente determinado por las glucemias G2h y no solamente por las glucemias de ayuno. Esto, junto a otras evidencias en las que se demuestra que las anomalías glucémicas en el periodo postprandial estarían involucradas en el desarrollo de enfermedades cardiovasculares (ECV) en DM, jerarquizan a la TTOG como una herramienta sumamente útil para el diag- nóstico precoz de alteraciones del metabolismo hidrocarbonado. La G2h aparece como un marcador de riesgo independiente para ECV. Por otra parte, queda claramente establecido que si se siguiera el criterio de la ADA para diagnóstico de DM se subdiagnosticaría el $30 \%$ de los casos de DM aproximadamente. Un diagnostico más precoz de DM o IGT permitiría tomar una serie de conductas terapéuticas que podrían evitar o enlentecer el desarrollo de ECV, principal causa de muerte en estos pacientes.

\section{Dr. León E. Litwak [ Servicio de Endocrinología, Metabolismo, y Medicina Nuclear. Hospital Italiano de Buenos Aires ]}

\section{Referencias}

Balkau B. New diagnostic criteria for diabetes and mortality in older adults. DECODE Study Group. European Diabetes Epidemiology Group. Lancet. 1999; 353:68-9.

Consequences of the new diagnostic criteria for diabetes in older men and women. DECODE Study (Diabetes Epidemiology: Collaborative Analysis of Diagnostic Criteria in Europe). Diabetes Care. 1999 Oct;22(10):1667-71

Will new diagnostic criteria for diabetes mellitus change phenotype of patients with diabetes? Reanalysis of European epidemiological data. DECODE Study Group on behalf of the European. Diabetes Epidemiology Study Group. BMJ. 1998;317:371-5 